Rev. Int. Contam. Ambie. 33 (1) 37-47, 2017

DOI: 10.20937/RICA.2017.33.01.03

\title{
EVALUACIÓN DE TRES SISTEMAS DE HUMEDALES HÍBRIDOS A ESCALA PILOTO PARA LA REMOCIÓN DE NITRÓGENO
}

\author{
Ángeles Xitlalli TORRES BOJORGES, Noemy Alejandra HERNÁNDEZ RAZO, \\ Airam Aseret FAUSTO URQUIETA y Florentina ZURITA MARTÍNEZ*
}

Laboratorio de Calidad Ambiental. Centro Universitario de la Ciénega. Av. Universidad 1115. Col. Lindavista. Ocotlán, Jalisco. México. C.P. 47820

*Autor para correspondencia: fzurita2001@yahoo.com

(Recibido agosto 2015; aceptado mayo 2016)

Palabras clave: nitrógeno amoniacal, nitrógeno orgánico, nitrato, humedales verticales, Canna indica, Strelitzia reginae

\section{RESUMEN}

En este estudio se evaluaron y compararon tres sistemas de humedales híbridos para la remoción de nitrógeno total $\left(\mathrm{N}_{\text {total }}\right)$ durante dos años. Se cuantificó el contenido de cada uno de los componentes nitrogenados: nitrógeno amoniacal $\left(\mathrm{N}_{-} \mathrm{NH}_{4}{ }^{+}\right)$, nitrato $\left(\mathrm{NO}_{3}{ }^{-}\right)$ y nitrógeno orgánico $\left(\mathrm{N}_{\text {Org }}\right.$ ). El primer sistema (I) estuvo compuesto de un humedal subsuperficial de flujo horizontal (HSSFH), seguido de una laguna de estabilización (LE) aerobia; el segundo (II) por HSSFH seguido de un humedal subsuperficial de flujo vertical (HSSFV); y el tercero (III), por HSSFV seguido de HSSFH. Los tres sistemas fueron muy efectivos para la nitrificación. Se registraron incrementos elevados en la concentración de $\mathrm{NO}_{3}{ }^{-}$en los efluentes y eficiencias altas en la remoción de $\mathrm{N}_{-} \mathrm{NH}_{4}{ }^{+}$. Estas eficiencias fueron de $76.2 \%$ y $78.7 \%$, en el sistema I; de $87.4 \%$ y $92.5 \%$ en el sistema II y de $82.6 \%$ y $97.2 \%$ en el sistema III, en el primer y segundo año, respectivamente. Respecto a la remoción de $\mathrm{N}_{\text {total }}$, el sistema I (HSSFH-LE) fue el más efectivo $(\mathrm{p}<0.05)$ con remociones de $59.4 \%$ y $57.7 \%$ en los años 1 y 2 , respectivamente. En los sistemas II (HSSFH-HSSFV) y III (HSSFV-HSSFH) las remociones fueron de $21.2 \%$ y $34.4 \%$, y de $25.0 \%$ y $36.3 \%$, respectivamente. La baja remoción de $\mathrm{N}_{\text {total }}$ en los sistemas II y III, aparentemente se debió a una baja tasa de desnitrificación ante la ausencia de zonas anóxicas/anaerobias más extensas como resultado de la baja profundidad de los HSSFH y de las características del medio filtrante, tales como su distribución de tamaño de partícula (tezontle molido) y su elevada porosidad.

Key words: ammonium, organic nitrogen, nitrate, vertical wetlands, Canna indica, Strelitzia reginae

\begin{abstract}
In this two-year study, three hybrid constructed wetlands were evaluated and compared for Total-N removal, quantifying the content of each of the nitrogen compounds: Ammonium $\left(\mathrm{NH}_{4}^{+}\right)$, nitrate $\left(\mathrm{NO}_{3}^{-}\right)$and organic nitrogen $(\mathrm{Org}-\mathrm{N})$. The first system (I) consisted of a subsurface flow horizontal wetland (SSFHW) followed by an aerobic stabilization pond (SP); the second one (II) consisted of a SSFHW followed by a subsurface flow vertical wetland (SSFVW); and the third one (III) was configured with a SSFVW followed by a SSFHW. The three systems were very effective for nitrification,
\end{abstract}


reaching a high increase in nitrate concentrations in the effluents and high efficiencies in the $\mathrm{N}^{-\mathrm{NH}_{4}}{ }^{+}$removal. These efficiencies were $76.2 \%$ and $78.7 \%$, in the system I; $87.4 \%$ and $92.5 \%$ in the system II and $82.6 \%$ and $97.2 \%$ in the system III, during the first and the second year, respectively. With regard to the removal of Total-N, the system I (SSFHW-SP) was the most effective $(\mathrm{p}<0.05)$ with removal of $59.4 \%$ and $57.7 \%$ in the first and the second year, respectively. In the systems II (SSFHW-SSFVW) and III (SSFVW-SSFHW), the removals were $21.2 \%$ and $34.4 \%$, and $25.0 \%$ and $36.3 \%$, respectively for the first and the second year. The low removal of Total-N in the systems II and III, apparently were due to a low rate of denitrification because of the absence of a larger anoxic/anaerobic area; as a result of the shallow depth of the SSFHWs and the characteristics of the filter medium such as its grain size distribution (ground tezontle) and high porosity.

\section{INTRODUCCIÓN}

En las aguas residuales municipales, el nitrógeno se encuentra presente esencialmente como nitrógeno orgánico $\left(\mathrm{N}_{\mathrm{Org}}\right)$, nitrógeno amoniacal $\left(\mathrm{N}^{-\mathrm{NH}_{4}}{ }^{+}\right)$y nitrato $\left(\mathrm{NO}_{3}{ }^{-}\right)$(Metcalf y Eddy 2003). Para la remoción total del nitrógeno, se requieren diversas transformaciones químicas que precisan de condiciones físicas y químicas distintas, de modo que generalmente las tasas de remoción de nitrógeno en los sistemas biológicos convencionales son inferiores a 10-20\% (Halling-Sorensen y Jorgensen 1993), a menos que se diseñen con el objetivo de remover nitrógeno. Por ejemplo, en el sistema de lodos activados se logra una remoción de $\mathrm{N}^{-\mathrm{NH}_{4}}{ }^{+}>50 \%$, mediante su transformación a $\mathrm{NO}_{3}{ }^{-}$, cuya remoción a su vez, es prácticamente nula (Metcalf y Eddy 2003). Sin embargo, en los diferentes tipos de humedales de tratamiento, se llevan a cabo las complejas reacciones de transformación del nitrógeno como son la nitrificación, desnitrificación, amonificación, reducción asimilativa de $\mathrm{NO}_{3}^{-}$, entre otras, por lo que se tienen mayores posibilidades de removerlo.

En los humedales de tratamiento, el primer paso para la remoción de $\mathrm{N}_{\text {total, }}$ es la transformación del $\mathrm{N}_{\text {Org }}$ a N-NH ${ }_{4}^{+}$(amonificación) mediante microorganismos aerobios o anaerobios, aunque las reacciones son más lentas en condiciones anaerobias (Kadlec y Knight 1996). El N-NH ${ }_{4}^{+}$formado o presente en el influente, puede seguir diversas rutas. Puede ser asimilado por las raíces de las plantas o por microorganismos anaerobios y transformarse de nuevo a $\mathrm{N}_{\text {Org. }}$. Si el pH es superior a 8 , el amonio se transformará a amoniaco y una parte se volatilizará del sistema, también puede ser inmovilizado mediante intercambio iónico con el medio filtrante (Mitsch y Gosselink 2007). Sin embargo, la conversión a $\mathrm{NO}_{3}{ }^{-}$ bajo condiciones aerobias por las Nitrosomonas y Nitrobacter, es el mecanismo principal que reduce la concentración de $\mathrm{N}^{-\mathrm{NH}_{4}}{ }^{+}$en los humedales de tratamiento (Vymazal y Kröpfelová 2008). El último paso es la remoción del $\mathrm{NO}_{3}{ }^{-}$del sistema, el cual se lleva a cabo por dos vías, la primera es la asimilación por las plantas, mediante el ingreso del $\mathrm{NO}_{3}{ }^{-}$a las raíces por difusión o con el flujo de agua (Paul y Clark 1996). La segunda vía es la desnitrificación, que se favorece en condiciones anóxicas/anaerobias. A través de ella, el $\mathrm{NO}_{3}{ }^{-}$se transforma a óxido nítrico (NO), a óxido nitroso $\left(\mathrm{N}_{2} \mathrm{O}\right)$ y finalmente a nitrógeno gas $\left(\mathrm{N}_{2}\right)$ (Kadlec y Knight 1996, Vymazal 2007). También se ha encontrado que el $\mathrm{NO}_{3}{ }^{-}$puede sufrir una reconversión a N-NH${ }_{4}^{+}$(reducción desasimilatoria a amoniaco) en ambientes donde existe un exceso de materia orgánica en relación con el $\mathrm{NO}_{3}{ }^{-}$(Kadlec y Knight 1996) y puede ser realizada por bacterias anaeróbicas, aeróbicas o facultativas (Mitsch y Gosselink 2007).

Las transformaciones del nitrógeno que predominan dependen sobre todo del tipo de humedal, como puede ser de flujo horizontal o vertical. En los humedales de flujo horizontal predominan las condiciones anóxicas/anaerobias debido a la saturación permanente del medio, por lo que proporcionan condiciones propicias para la desnitrificación si hay presencia de materia orgánica (Vymazal 2013). En contraste, en los humedales de flujo vertical predominan las condiciones aerobias, favorables para la nitrificación, debido a que la alimentación intermitente facilita la difusión del oxígeno en el lecho (Vymazal 2007). Por lo tanto, se requiere de la combinación de diferentes tipos de humedales instalados en serie, conocidos como humedales híbridos, para promover la remoción del $\mathrm{N}_{\text {total }}$ (Vymazal y Kröpfelová 2011). Tales sistemas se han estudiado y en general, han mostrado ser más efectivos que los sistemas de una sola etapa para la remoción de $\mathrm{N}_{\text {total }}$. Sin embargo, muchos de estos estudios se han realizado en regiones de climas templados y fríos del norte y centro de 
Europa (Vymazal 2013) y muy pocos en los climas tropicales y subtropicales de América Latina.

En este estudio se evaluaron tres sistemas de humedales híbridos (SHI) a escala piloto en un clima subtropical, con los objetivos de evaluar y comparar sus eficiencias para la remoción de $\mathrm{N}_{\text {total }}$ en dos periodos de experimentación de un año de duración cada uno.

\section{MATERIALES Y MÉTODOS}

\section{Descripción de la unidad experimental}

La descripción detallada de los sistemas estudiados, se encuentra en Zurita y White (2014). Los humedales subsuperficiales de flujo horizontal (HSSFH), humedales subsuperficiales de flujo vertical (HSSFV) y las lagunas de estabilización (LE) tuvieron las siguientes dimensiones: $120 \mathrm{~cm} \times 40$ $\mathrm{cm} \times 50 \mathrm{~cm}(\mathrm{~L} \times \mathrm{A} \times \mathrm{H}) ; 48 \mathrm{~cm} \times 48 \mathrm{~cm} \times 110 \mathrm{~cm}$ $(\mathrm{L} \times \mathrm{A} \times \mathrm{H})$ y $70 \mathrm{~cm} \times 70 \mathrm{~cm} \times 70 \mathrm{~cm}(\mathrm{~L} \times \mathrm{A} \times \mathrm{H})$, respectivamente. En la figura 1 se esquematizan los tres sistemas de tratamiento, evaluados por duplicado. El sistema I, estuvo conformado por un HSSFH (1 ${ }^{\text {a }}$ etapa) seguido de una LE aerobia ( $2^{\text {a }}$ etapa). Los HSSFH se alimentaron en forma continua, con un tiempo de retención hidráulico (TRH) teórico de 3 días. El efluente de las HSSFH fluyó por gravedad hacia las LE. Similar al sistema I, el sistema II estuvo compuesto por un HSSFH como primera etapa seguido por un HSSFV como segunda etapa. Los HSSFH operaron de la misma manera como en el sistema I, pero su efluente se recogió en un depósito y se bombeó de forma intermitente cada $2 \mathrm{~h}$ sobre el sustrato de los HSSVF. El sistema III estuvo in-

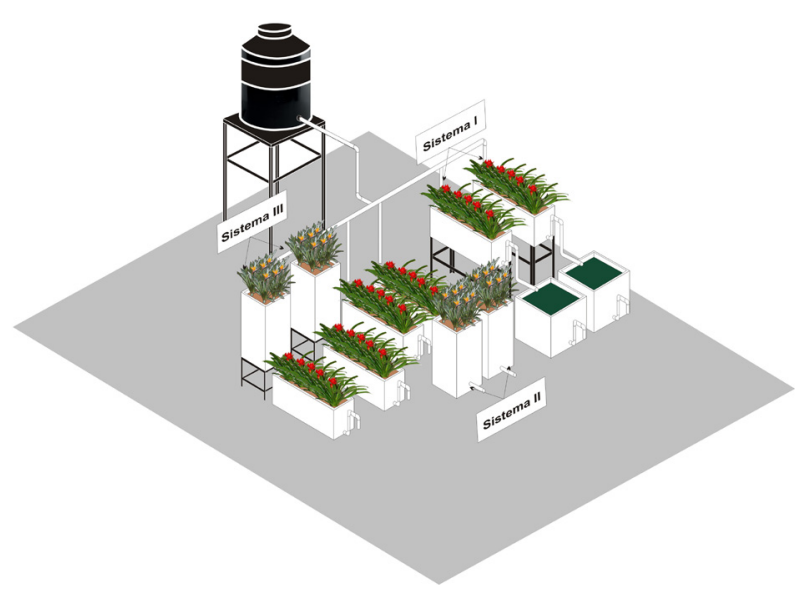

Fig. 1. Humedales híbridos empleados en este estudio (Zurita y White 2014) tegrado por un HSSFV seguido por un HSSFH. Los HSSFV se alimentaron de forma intermitente por medio de una bomba que fue programada para descargar $2.8 \mathrm{~L}$ cada $2 \mathrm{~h}$ sobre la superficie del sustrato, su efluente fluyó por gravedad hacia los HSSFH. En total, en el sistema se trató un caudal de $\sim 200 \mathrm{~L} / \mathrm{d}$ de agua residual que se distribuyó equitativamente en los tres sistemas.

En cada HSSFH se plantaron seis individuos de Zantedeschia aethiopica (de 25 a $30 \mathrm{~cm}$ de altura), mientras que en los HSSFV solamente se plantó un individuo adulto de Strelitzia reginae (especie ornamental de valor comercial ya probada en estudios previos) que permite la posibilidad de combinar el tratamiento de las aguas residuales con la producción de flores (Zurita et al. 2009). Después de 8 meses de experimentación, los individuos de Z. aethiopica fueron reemplazados con Canna indica debido a que la $Z$. aethiopica se secó durante la estación de estiaje caracterizada por una menor humedad relativa y temperaturas ambientales más elevadas. Durante el segundo año, Canna indica se mantuvo en los HSSFH. Como medio filtrante se utilizó tezontle con un diámetro efectivo de $\mathrm{d}_{10}=0.645 \mathrm{~mm}$ y de $\mathrm{d}_{60}=2.3 \mathrm{~mm}$ y un coeficiente de uniformidad (CU) de 3.6.

\section{Parámetros de calidad del agua}

Los sistemas se alimentaron desde el inicio con agua residual generada en el campus universitario (cuadro I). Estas aguas se bombearon desde un colector hacia un depósito adaptado, con el propósito de disminuir la concentración de sólidos suspendidos totales por sedimentación. Se proporcionó un periodo de estabilización de cuatro meses, antes de iniciar el monitoreo semanal, que se realizó durante ocho meses en el primer año de estudio. En el segundo año, el monitoreo se realizó cada 15 días. Se tomaron muestras de agua residual en el influente y efluente de cada etapa en los tres SHI para realizar determinaciones de $\mathrm{N}_{\mathrm{Org}}, \mathrm{N}_{-} \mathrm{NH}_{4}{ }^{+}$y $\mathrm{NO}_{3}{ }^{-}$. En forma complementaria se midieron, la demanda química de oxígeno, fósforo total, sólidos suspendidos totales, oxígeno disuelto, $\mathrm{pH}$ y conductividad en el influente, con el objeto de caracterizar adecuadamente el agua residual cruda. Los parámetros de calidad del agua se determinaron de acuerdo con las técnicas descritas en los Métodos Estándar para el Análisis de Aguas y Aguas Residuales (APHA, AWWA, WEF, 2005). Se utilizaron un potenciómetro (Thermo Scientific 3 Star) y un medidor de oxígeno disuelto (Orion 3 Star Thermo Electron) para medir el $\mathrm{pH}$ y la conductividad, y el oxígeno disuelto, respectivamente. 
Las muestras se analizaron inmediatamente después de ser tomadas en el Laboratorio de Calidad Ambiental del campus universitario.

\section{Análisis estadístico}

Se utilizó un diseño de bloques completos aleatorizados para analizar los datos a lo largo del tiempo. Asimismo, se realizaron análisis de varianza (ANDEVA) multifactorial con el paquete Statgraphics Centurion XVI. Cuando se observaron diferencias significativas entre los tratamientos (valor de $p<$ 0.05 ) al realizar el ANDEVA, se efectuaron pruebas de rangos múltiples con el método de la diferencia mínima significativa (LSD, por sus siglas en inglés).

\section{Cálculos}

El porcentaje de remoción en cada etapa de tratamiento o sistema global se obtuvo a partir de las concentraciones promedio del influente $\left(\mathrm{C}_{\text {inf }}\right)$ y del efluente $\left(\mathrm{C}_{\mathrm{eff}}\right)$ correspondiente (ecuación 1):

$\%$ Remoción $=\left(\frac{C_{\text {inf }}-C_{\text {efl }}}{C_{\text {inf }}}\right) \times 100$

\section{RESULTADOS Y DISCUSIÓN}

En el cuadro I se muestran las características físicas y químicas del agua residual empleada en este estudio, durante los dos periodos de evaluación. La baja concentración de materia orgánica, medida como demanda química de oxígeno (DQO) y de sólidos suspendidos totales permite clasificar a este influente como de tipo débil de acuerdo con Metcalf y Eddy (2003). Sin embargo, la concentración de $\mathrm{N}_{\text {total }}$, en particular en la forma de $\mathrm{N}_{-} \mathrm{NH}_{4}{ }^{+}$(cuadro II), en los dos años de estudio, fue entre dos y casi tres veces la

CUADRO I. CARACTERÍSTICAS DEL AGUA RESIDUAL UTILIZADA DURANTE LOS DOS PERIODOS DE EVALUACIÓN. VALORES PROMEDIO \pm DESVIACIÓN ESTÁNDAR

\begin{tabular}{|c|c|c|}
\hline Parámetro & Año 1 & Año 2 \\
\hline $\begin{array}{l}\text { Demanda química de } \\
\text { oxígeno }(\mathrm{mg} / \mathrm{L})\end{array}$ & $273.5 \pm 145.5$ & $235.2 \pm 166.8$ \\
\hline Fósforo total $(\mathrm{mg} / \mathrm{L})$ & $12.4 \pm \quad 5.8$ & $8.1 \pm \quad 3.4$ \\
\hline $\begin{array}{l}\text { Sólidos suspendidos } \\
\text { totales }(\mathrm{mg} / \mathrm{L})\end{array}$ & $61.8 \pm 38.0$ & $65.6 \pm 50.4$ \\
\hline Oxígeno disuelto $(\mathrm{mg} / \mathrm{L})$ & $1.5 \pm \quad 0.97$ & $3.0 \pm \quad 2.4$ \\
\hline $\mathrm{pH}$ & $8.2 \pm$ & $8.2 \pm$ \\
\hline Conductividad $(\mu \mathrm{S} / \mathrm{cm})$ & $1797 \pm 810$ & $1418 \pm 492$ \\
\hline
\end{tabular}

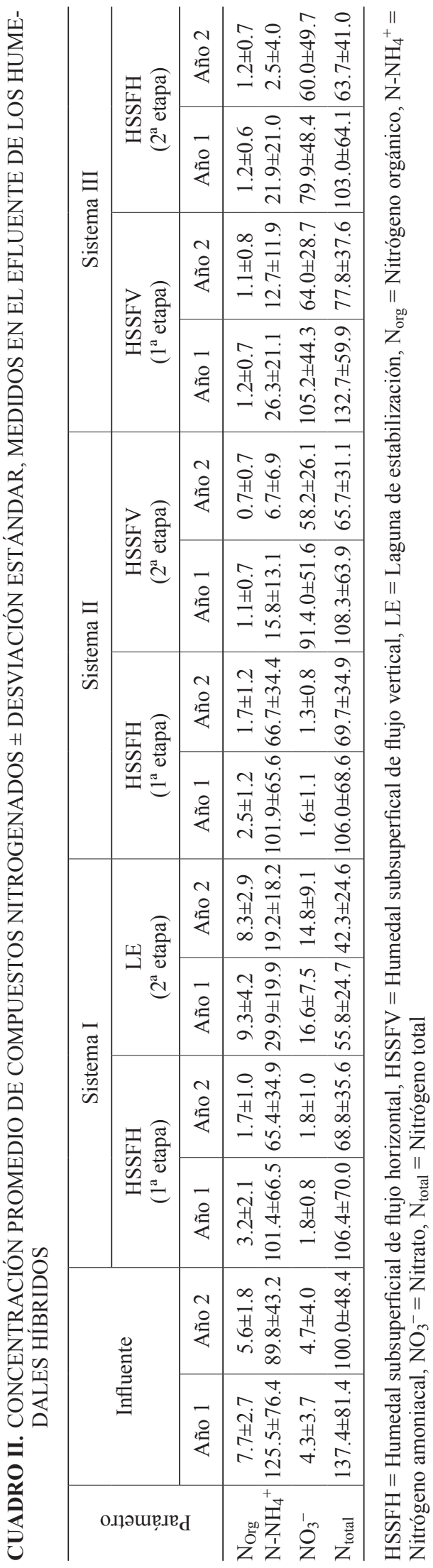


concentración de $45 \mathrm{mg} / \mathrm{L}$ de N-NH${ }_{4}^{+}$definidos para un agua residual doméstica clasificada como de concentración fuerte (Metcalf y Eddy 2003). Respecto al fósforo total, en los dos periodos de evaluación, su concentración fue similar a lo encontrado en aguas residuales clasificadas como de concentración fuerte de acuerdo con Metcalf y Eddy (2003). Estas elevadas concentraciones contribuyeron al buen desarrollo de las especies vegetales empleadas, ya que el fósforo es un macronutriente que las plantas requieren en grandes cantidades, ubicándose sólo debajo del nitrógeno. De esta manera, este parámetro de calidad del agua residual, probablemente influyó en los resultados de remoción de nitrógeno que se discutirán más adelante, ya que permitió un buen desarrollo de las especies vegetales, contribuyendo de manera positiva en las eficiencias de remoción (Cui et al. 2010). En contraste, la relativamente elevada conductividad eléctrica del agua residual utilizada, probablemente contribuyó a la desaparición de la Z. aethiopica en los meses de estiaje mencionado anteriormente, ya que esta especie se había evaluado previamente con aguas residuales con características similares, pero con una menor conductividad eléctrica y se había desarrollado vigorosamente (Zurita et al. 2009). Sin embargo, $C$. indica no mostró signos de afectación alguno. Finalmente, el oxígeno disuelto y el $\mathrm{pH}$, son dos parámetros que tienen influencia en el proceso de nitrificación, paso inicial para la remoción de nitrógeno cuando la forma predominante es la amoniacal. En este estudio, la presencia de cierto contenido de oxígeno disuelto en el influente, seguramente favoreció tal proceso en los HSSFH ubicados como primera etapa. De manera similar, los valores de $\mathrm{pH}$ fueron propicios para la nitrificación al encontrarse en el rango óptimo (7.2-9) para tal proceso (Kadlec y Wallace 2009).

En el cuadro II, se muestra la concentración de las diferentes especies de nitrógeno en cada uno de los sistemas híbridos, durante los dos periodos de evaluación. A partir de las concentraciones promedio, determinadas para cada especie de nitrógeno, se calculó el porcentaje de remoción en cada una de las etapas de los SHI, así como de manera global.

\section{Humedal híbrido I (HSSFH-LE)}

Nitrógeno orgánico. Durante el primer año de operación, la concentración de $\mathrm{N}_{\text {Org }}$ se redujo significativamente en los HSSFH y se incrementó en las LE (Cuadro II, Fig. 2A; p < 0.05); alcanzándose una remoción de $58.4 \%$ y un incremento de $190.7 \%$, respectivamente. Durante el segundo año, el comportamiento fue similar (Fig. 2 B; p $<0.05$ ) y la remoción e incremento, alcanzados fueron de $70.0 \%$ y $388.0 \%$ en la primera y segunda etapa, respectivamente. Estos resultados sugieren que en la primera etapa, el $\mathrm{N}_{\text {Org }}$ presente en el agua residual se redujo a N-NH${ }_{4}^{+}$y posteriormente, en la segunda etapa (LE), las algas presentes transformaron el $\mathrm{N}^{-\mathrm{NH}_{4}}{ }^{+}$ y $\mathrm{NO}_{3}{ }^{-}$, a $\mathrm{N}_{\text {Org }}$ (Park et al. 2011). De esta manera, al tener lugar una alta transformación de estas formas nitrogenadas, a $\mathrm{N}_{\text {Org }}$, de manera global se registraron incrementos (en vez de remoción) de $21 \%$ y $48 \%$, en el primer y segundo año, respectivamente, sin diferencia entre ellas $(p>0.05)$. Estos resultados son característicos de lagunas con presencia de algas, ya que las algas microscópicas o macroscópicas, requieren grandes cantidades de $\mathrm{N}$ y $\mathrm{P}(45-60 \%$ en peso seco) para la formación de proteínas; razón por la cual se ha discutido de manera abundante en la literatura, la necesidad de cosechar las algas para incrementar la remoción de nutrientes (Rawat et al. 2011).

Por otra parte, se observó un incremento significativo en la eficiencia de los HSSFH durante el segundo año $(\mathrm{p}<0.05)$ para la remoción de $\mathrm{N}_{\text {Org. }}$. Esto sugiere
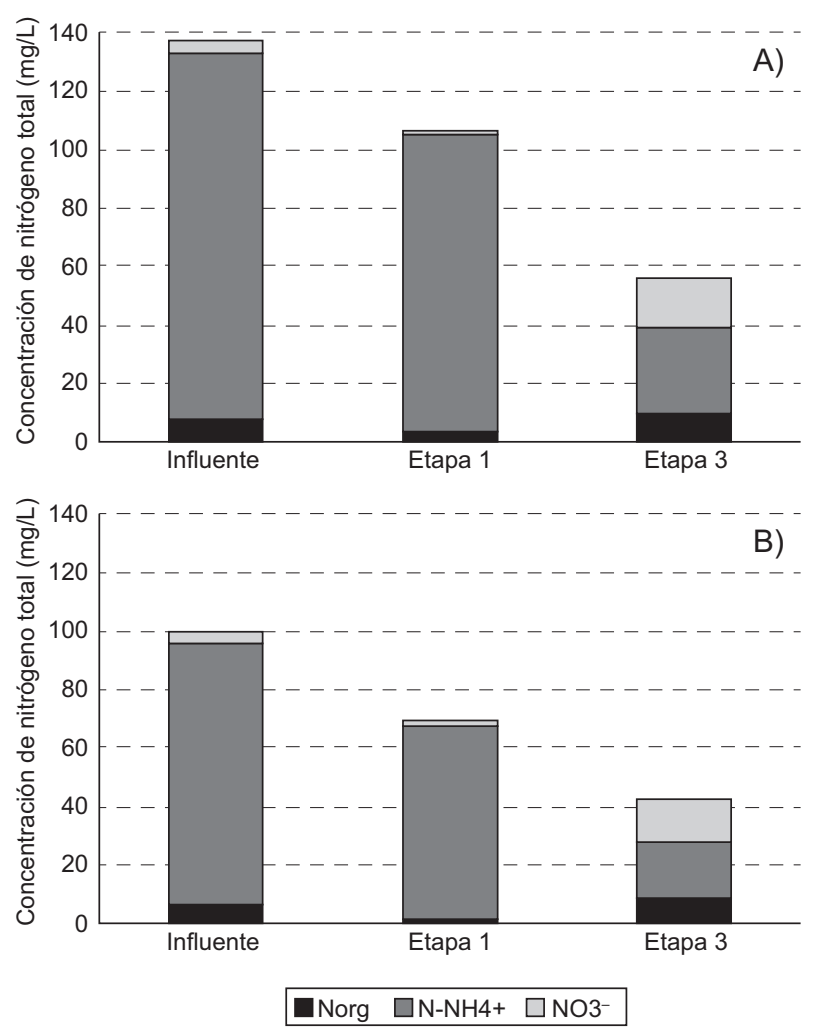

Fig 2. Concentraciones promedio de nitrógeno total en el sistema híbrido I, durante A) el primer año de operación, B) durante el segundo año de operación. $\mathrm{N}_{\text {org }}=$ Nitrógeno orgánico, $\mathrm{N}-\mathrm{NH}_{4}{ }^{+}=$Nitrógeno amoniacal, $\mathrm{NO}_{3}{ }^{-}=\mathrm{Ni}-$ trato 
una mayor capacidad de los HSSFH de este sistema, para la amonificación que se lleva a cabo con mayor rapidez en condiciones más oxidadas; probablemente, estas condiciones pudieron haber sido propiciadas por una mayor aportación de oxígeno por las plantas durante el segundo año, ya que en este periodo se observó un crecimiento vigoroso de las mismas y una cobertura vegetal completa por la $C$. indica.

Nitrógeno amoniacal. En ambos periodos de operación, se registró una disminución significativa del N-NH${ }_{4}^{+}$en los HSSFH y nuevamente en las LE (Cuadro II, Fig. 2A; $>$ <.05). De esta manera, en el primer año, las remociones fueron de $19.1 \%$ y 70.6 $\%$, respectivamente; mientras que, en el segundo, las remociones fueron de $27.1 \%$ y $70.7 \%$, respectivamente; sin diferencia significativa entre los sistemas durante los dos periodos evaluados $(p>0.05)$. La remoción global de $\mathrm{N}^{-\mathrm{NH}_{4}}{ }^{+}$, en el primer año fue de $76.2 \%$, mientras que en el segundo año fue de 78.7 $\%$, sin diferencia significativa $(\mathrm{p}>0.05)$. Resultados similares fueron reportados por Rivas et al. (2011) al evaluar un sistema híbrido similar (HSSFH - laguna de maduración) para tratar agua residual real. Los autores obtuvieron una remoción de $\mathrm{N}-\mathrm{NH}_{4}{ }^{+}$de $7 \%$, en el HSSFH y de $64 \%$ en la laguna de maduración. En las lagunas de estabilización, el crecimiento y metabolismo de las algas presentes ocasiona un aumento en el valor de $\mathrm{pH}$ durante el día, lo que favorece la volatilización del $\mathrm{N}-\mathrm{NH}_{4}{ }_{4}$, sobre todo a un $\mathrm{pH}$ por encima de 8. En contraste, durante la fase nocturna, la liberación de $\mathrm{CO}_{2}$ por la respiración de las algas, conduce a la disminución de $\mathrm{pH}$, imposibilitando la pérdida de $\mathrm{N}-\mathrm{NH}_{4}{ }^{+}$por volatilización (Mitsch y Gosselink 2007, Vymazal 2007). La volatilización de $\mathrm{N}^{-\mathrm{NH}_{4}}{ }^{+}$en lagunas, como resultado de las elevaciones diurnas de $\mathrm{pH}$, también fue evidenciado por Rivas et al. (2011).

Nitrato. Durante los dos años de operación, la concentración de $\mathrm{NO}_{3}{ }^{-}$disminuyó significativamente en los HSSFH, pero se incrementó en las LE (Cuadro II, Fig. 2A y 2B; $p<0.05)$ al prevalecer condiciones más oxidadas, favorables para la nitrificación (Buss et al. 2004). Así, durante el primer año, la remoción en los HSSFH fue de $58.1 \%$ y el incremento en los HSSFV, de $822.2 \%$, respectivamente; mientras que en el segundo año, estos valores fueron de $61.7 \%$ y de $722.2 \%$, respectivamente. No se encontró diferencia significativa en los HSSFH ni en las LE durante los dos periodos $(p>0.05)$. Ante la alta transformación del $\mathrm{N}^{-\mathrm{NH}_{4}}{ }^{+}$a $\mathrm{NO}_{3}{ }^{-}$en las LE, se registraron incrementos globales de $\mathrm{NO}_{3}{ }^{-}$(en lugar de remoción) de $286 \%$ y $215 \%$, en los años 1 y 2 , respectivamente.

Nitrógeno Total. En este sistema, la concentración de $\mathrm{N}_{\text {total }}$ disminuyó significativamente en los HSSFH y nuevamente en las LE ( $p>0.05)$ y tal comportamiento se registró en los dos años (Cuadro II, Fig. 2A y 2B). Las remociones logradas, fueron de $22.6 \%$ y $47.6 \%$, respectivamente, durante el primer año; en tanto que en el segundo año, fueron de 31.2 $\%$ y de $38.5 \%$, respectivamente. Los porcentajes de remoción logrados en los HSSFH, son inferiores al valor promedio de $42 \%$ de 137 sistemas alrededor del mundo, reportado por Vymazal $(2005,2007)$. Es probable que estos resultados más bajos, puedan deberse a la poca profundidad de los HSSFH $(0.40 \mathrm{~m})$, en comparación con las profundidades generalmente reportadas en la literatura (0.6-0.8 m) (Vymazal 2005), así como a la distribución del tamaño de partícula del medio filtrante utilizado, que en conjunto impidieron la formación de zonas anóxicas/anaerobias más amplias, deseables para la desnitrificación. Los HSSFH poco profundos $(30-40 \mathrm{~cm})$ con un medio filtrante fino, tienden a exhibir condiciones más aeróbicas que los de mayor profundidad, debido a que un mayor volumen de agua entra en contacto con la zona de raíces de las macrófitas (Morató et al. 2014). Esto se ha confirmado al encontrar concentraciones de oxígeno disuelto y valores de potencial redox más elevados, en capas superiores en comparación con las inferiores, en HSSFH de más de $60 \mathrm{~cm}$ (Ding et al. 2014). Las remociones globales fueron de $59.4 \%$ y de $57.7 \%$, respectivamente, sin diferencia significativa en los dos periodos $(\mathrm{p}>0.05)$. Tales resultados, son similares a los reportados por Rivas et al. (2011) al evaluar un sistema híbrido HSSFH-Laguna de maduración.

\section{Humedal híbrido II (HSSFH-HSSFV)}

Nitrógeno orgánico. Para ambos años de operación, la concentración del $\mathrm{N}_{\mathrm{Org}}$, disminuyó significativamente en los HSSFH y nuevamente, en los HSSFV (Cuadro II, Fig. 3A y 3B; $p<0.05$ ). Las remociones alcanzadas en el primer año fueron de $67.4 \%$ y $56.0 \%$, respectivamente; mientras que, en el segundo año, fueron de $69.6 \%$ y $58.8 \%$; sin diferencia entre los periodos $(\mathrm{p}>0.05)$. Las remociones globales fueron de $85.7 \%$ y $87.5 \%$, respectivamente $(\mathrm{p}>0.05)$. De esta manera, este SHI fue superior al sistema I para la remoción de $\mathrm{N}_{\text {Org }}$, al incluir un componente vertical.

Nitrógeno amoniacal. Similar al $\mathrm{N}_{\mathrm{Org}}$, la concentración de $\mathrm{N}^{-\mathrm{NH}_{4}}{ }^{+}$se redujo significativamente en los HSSFH y nuevamente en los HSSFV en los dos periodos de evaluación (Cuadro II, Fig. 3A y 3B; $\mathrm{p}<0.05$ ). Las remociones fueron de $18.8 \%$ y de $84.5 \%$, respectivamente en el primer año; en tanto que, en el segundo año, fueron de 25.6 y $90.0 \%$, respectivamente. Acorde con lo esperado, los HSSFV 

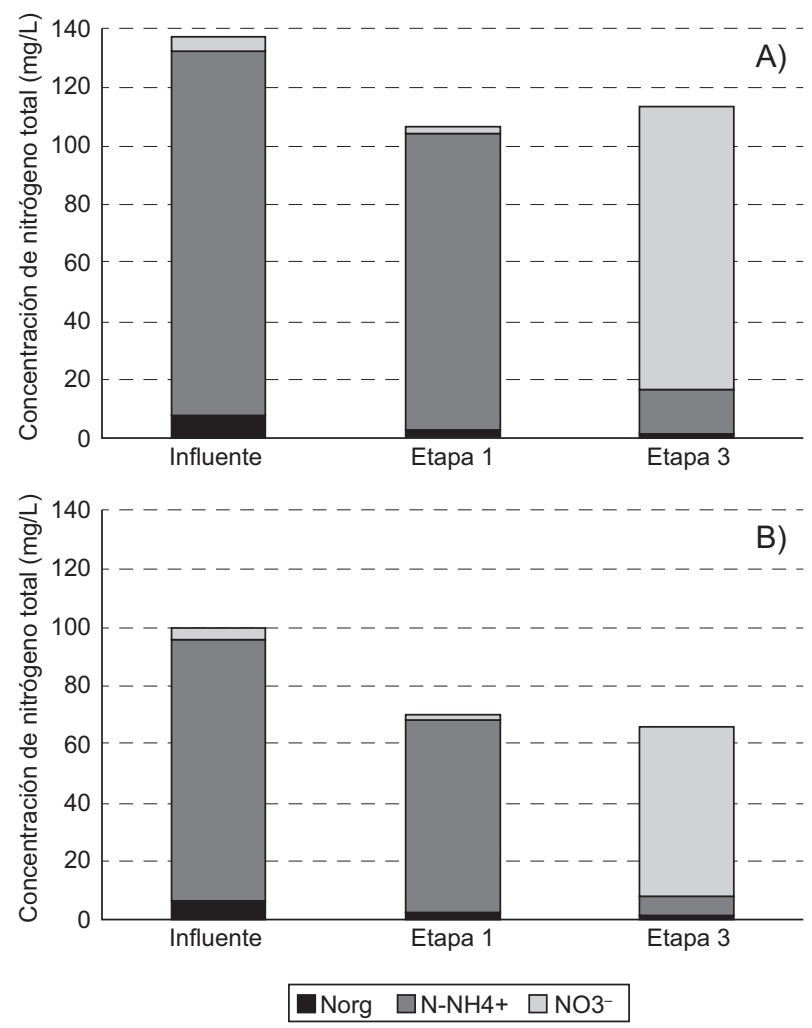

Fig. 3. Concentraciones promedio de nitrógeno total en el sistema híbrido II, durante A) el primer año de operación, B) durante el segundo año de operación. $\mathrm{N}_{\text {org }}=$ Nitrógeno orgánico, $\mathrm{N}_{-} \mathrm{NH}_{4}{ }^{+}=$Nitrógeno amoniacal, $\mathrm{NO}_{3}{ }^{-}=\mathrm{Ni}-$ trato

fueron efectivos para nitrificar debido a su elevada capacidad de transferencia de oxígeno (Cui et al.2010) y su eficiencia se incrementó significativamente en el segundo año $(\mathrm{p}<0.05)$. Es probable que, durante el segundo año, la captación de amonio por la $S$. reginae, haya contribuido a este incremento, ya que en este periodo se observó una mayor producción de hojas y flores en esta especie. Con respecto a los HSSFH, aunque el incremento en su eficiencia en el segundo año, no fue significativo (al igual que en el sistema I), sí muestra una tendencia hacia un valor más alto; probablemente debido a la mayor cobertura total vegetativa por la $C$. indica que en este periodo alcanzó una altura hasta de $2 \mathrm{~m}$, lo que seguramente provocó una mayor oxigenación de la rizósfera y una mayor captación del nitrógeno. De esta manera, la remoción global fue de $87.4 \%$ en el primer año, y se incrementó significativamente a $92.5 \%$ en el segundo año $(p>0.05)$. Tales resultados son superiores al $86 \%$ logrado en un sistema híbrido similar evaluado en un clima mediterráneo (HSSFV de $0.9 \mathrm{~m}$ con arena de 0-4mm;HSSFHde $0.7 \mathrm{~m}$ dealtura congravade $5-10 \mathrm{~mm}$; ambos plantados con Phragmites) (Massi 2007).
Nitrato. Al igual que en el sistema I, en los dos periodos, la concentración de $\mathrm{NO}_{3}{ }^{-}$disminuyó significativamente en los HSSFH y posteriormente se incrementó en los HSSFV (Cuadro II, Fig. 3A y 3B; $\mathbf{p}<$ 0.05). Así, las remociones fueron de $62.8 \%$ y $72.3 \%$ en los HSSFH en el primer y segundo año, respectivamente, sin diferencia significativa entre ellos $(\mathrm{p}>0.05)$. La remoción de $\mathrm{NO}_{3}{ }^{-}$en los HSSFH de los dos sistemas, concuerda con lo esperado, ya que las condiciones predominantes en este tipo de humedales como primera etapa (presencia de materia orgánica en el agua residual y bajas concentraciones de oxígeno disuelto), favorecen la desnitrificación. Por otra parte, tanto en los HSSFV como en los sistemas globales, se registraron incrementos en las concentraciones de $\mathrm{NO}_{3}{ }^{-}$debido a la alta capacidad de los HSSFV para la nitrificación. De esta forma, los incrementos en la concentración de $\mathrm{NO}_{3}{ }^{-}$en este sistema con respecto al influente, fueron de $2025 \%$ y $1138 \%$, en el primer y segundo año, respectivamente. El incremento en la concentración de $\mathrm{NO}_{3}{ }^{-}$en el efluente final indica de manera contundente que el mecanismo principal en la remoción de $\mathrm{N}_{-} \mathrm{NH}_{4}{ }^{+}$, fue la nitrificación (Sun et al. 2005).

Nitrógeno total. El comportamiento del $\mathrm{N}_{\text {total }}$ fue similar en los dos años (Cuadro II, Fig. 3A y 3B), la concentración disminuyó significativamente en los HSSFH $(\mathrm{p}<0.05)$ y se mantuvo sin cambio significativo en los HSSFV. Así, con base en las concentraciones promedio, la remoción fue de $22.8 \%$ en los HSSFH, durante el primer año; mientras que en el segundo año fue de $30.3 \%$. Finalmente, las remociones globales fueron de $21.2 \%$ y de $34.4 \%$, respectivamente; con un incremento significativo en el segundo año $(\mathrm{p}<0.05)$. En promedio, estos porcentajes de remoción son similares al $29 \%$ logrado en un sistema híbrido HSSFH-HSSFV (ambos con $0.8 \mathrm{~m}$ de altura) plantados con Phragmites australis y con un medio filtrante compuesto de mármol, piedra caliza, arena y grava (Ayaz et al. 2015).

\section{Humedal híbrido III (HSSFV-HSSFH)}

Nitrógeno orgánico. En este sistema, al igual que en el sistema II, el comportamiento fue similar en los dos periodos de evaluación (Cuadro II, Fig. 4A y 4B), la concentración de $\mathrm{N}_{\text {Org }}$ disminuyó significativamente en los HSSFV $(p<0.05)$ y posteriormente ya no cambió significativamente en los HSSFH. De esta manera, en el primer año, la remoción fue de $84.4 \%$ en los HSSFV. En tanto que, en el segundo año, fue de $80.4 \%$, sin diferencia significativa entre los dos periodos de estudio $(p>0.05)$. Las remociones globales fueron de $84.4 \%$ y $78.6 \%$, para el año 1 
y 2 , respectivamente, sin diferencia significativa $(\mathrm{p}>0.05)$.

Nitrógeno amoniacal. Durante el primer año de experimentación, la concentración de $\mathrm{N}_{-} \mathrm{NH}_{4}{ }^{+}$ disminuyó significativamente en los HSSFV ( $\mathrm{p}<$ 0.05 ), pero ya no cambió significativamente al pasar por los HSSFH (Cuadro II, Fig. 4A), la remoción alcanzada en el HSSFV fue de $79 \%$. Sin embargo, durante el segundo año, la concentración se redujo significativamente en los HSSFV y nuevamente en los HSSFH (Cuadro II, Fig. 4B; $p<0.05$ ), alcanzándose remociones de $85.9 \%$ y $80.3 \%$, respectivamente. La eficiencia de los HSSFV se incrementó significativamente durante el segundo año $(p<0.05)$, al igual que la eficiencia de los HSSFH que operaron como segunda etapa. El incremento en la eficiencia de los dos tipos de humedales puede atribuirse a una mayor madurez de los sistemas después de un año de operación, caracterizada por un desarrollo vigoroso de la vegetación. Fue muy notorio la producción de flores y hojas de $S$. reginae en los HSSFV y el crecimiento vigoroso con producción de tallos y flores de $C$. indica en los HSSFH. Este desarrollo de la vegetación pudo incrementar la remoción de $\mathrm{N}_{-} \mathrm{NH}_{4}{ }^{+}$ mediante la captación directa, tal como fue reportado por Cui et al. (2010) al evaluar HSSFV sin plantas y con $C$. Indica. Adicionalmente, las plantas también pudieron contribuir con la presencia de zonas más oxidadas favorables para la nitrificación sobre todo en los HSSFH. Por otra parte, y acorde con lo esperado, la capacidad de los HSSFV como primera etapa en este sistema, fue superior a la de los HSSFH ( $p<$ 0.05 ) de los sistemas I y II. Finalmente, la remoción global alcanzada, fue de $82.6 \%$ en el primer año, y similar al sistema II, se incrementó significativamente a $97.2 \%(p<0.05)$ en el segundo año. La remoción de $\mathrm{N}^{-} \mathrm{NH}_{4}{ }^{+}$lograda en el segundo año, se encuentra entre los más altos registrados en la literatura (Brix 2003, Masi y Martinuzzi 2007, Vymazal 2013).

Nitrato. Acorde con lo esperado, durante el primer año, la concentración de $\mathrm{NO}_{3}{ }^{-}$se incrementó significativamente en los HSSFV, en un $2346.5 \%$. Posteriormente se registró una reducción significativa en los HSSFH, con una remoción de $24.0 \%$ (Cuadro II, Fig. 4A). Respecto al segundo año, similar al primero, se registró un incremento de $261.7 \%$ en los HSSFV, en tanto que la concentración ya no cambió significativamente en los HSSFH ( $p>0.05)$. Como resultado final, los incrementos globales fueron de $1758.1 \%$ y de $1176.6 \%$, para el primer y segundo año, respectivamente. Probablemente durante el segundo año, las condiciones en los HSSFH fueron menos propicias para la desnitrificación al tener lugar la presencia de zonas menos anóxicas ante una mayor oxigenación del sistema por la mayor cobertura y desarrollo de las plantas.

Nitrógeno total. El comportamiento del $\mathrm{N}_{\text {total }}$ en el sistema III, fue diferente en los dos años de evaluación. En el primero, la concentración de $\mathrm{N}_{\text {total }}$ no se modificó en los HSSFV, pero sí disminuyó significativamente en los HSSFH $(\mathrm{p}<0.05)$, en el que la remoción fue de $22.4 \%$ (Cuadro II, Fig. 4A). Es frecuente encontrar una baja capacidad de HSSFV para la remoción de $\mathrm{N}_{\text {total }}$, ya que normalmente carecen de las condiciones físicas y químicas para que tenga lugar la desnitrificación. Por ejemplo, Langergraber et al. (2008) encontraron una remoción promedio de sólo $8 \%$ en HSSFV de $75 \mathrm{~cm}$ de altura con grava de 1-4 mm de diámetro. Por otro lado, Masi y Martinuzzi (2007) reportaron una remoción nula general de $\mathrm{N}_{\text {total }}$ en HSSFV en países mediterráneos. Respecto al segundo año, la concentración se redujo significativamente en los HSSFV y posteriormente, también en los HSSFH $(\mathrm{p}<0.05)$. Las remociones alcanzadas fueron de $22.2 \%$ y de $22.9 \%$, respectivamente
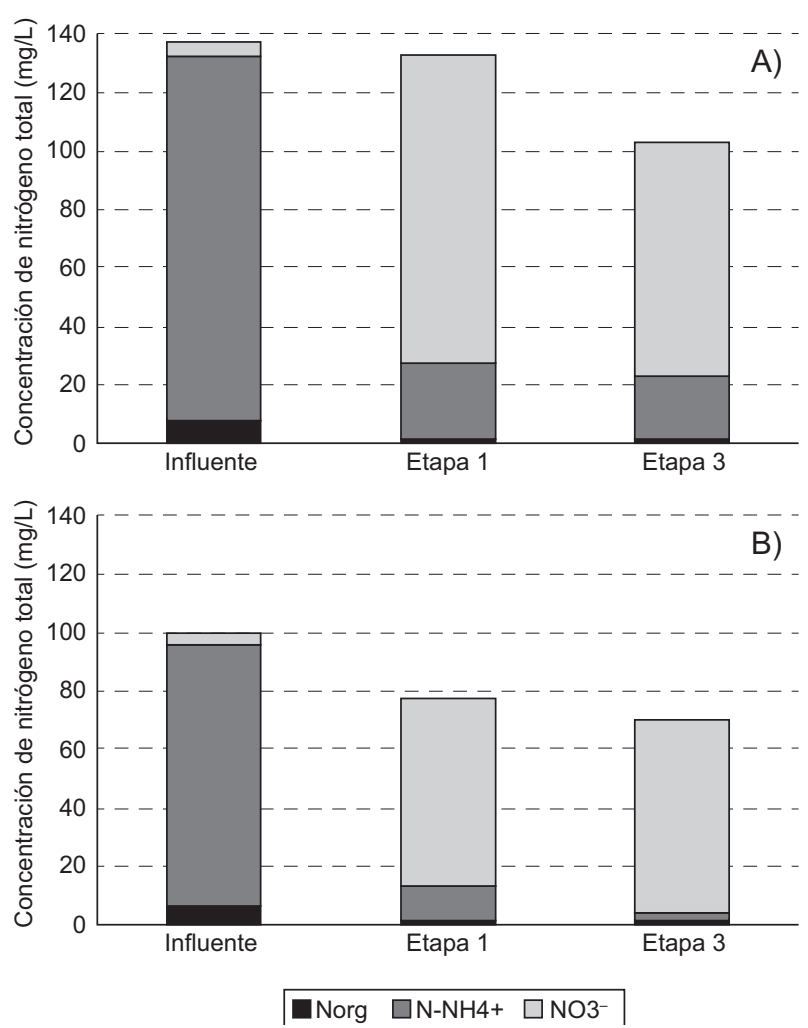

Fig. 4. Concentraciones promedio de nitrógeno total en el sistema híbrido III, durante A) el primer año de operación, B) durante el segundo año de operación. $\mathrm{N}_{\text {org }}=$ Nitrógeno orgánico, ${\mathrm{N}-\mathrm{NH}_{4}}^{+}=$Nitrógeno amoniacal, $\mathrm{NO}_{3}{ }^{-}=\mathrm{Ni}-$ trato 
(Cuadro II, Fig. 4B). El incremento en la capacidad de los HSSFV durante el segundo año, probablemente pudo deberse a la captación de $\mathrm{N}^{-\mathrm{NH}_{4}}{ }^{+} \mathrm{y} \mathrm{NO}_{3}{ }^{-}$por la vegetación ya bien establecida. Las remociones globales alcanzadas en los dos periodos, fueron de $25.0 \%$ y de $36.3 \%$ respectivamente, sin diferencia significativa $(\mathrm{p}>0.05)$.

\section{Comparación de los tres sistemas híbridos para la remoción de nitrógeno total}

Durante los dos periodos de operación, el sistema I (HSSFH-LE) fue el sistema más efectivo $(\mathrm{p}<0.05)$ para la remoción de $\mathrm{N}_{\text {total }}$, alcanzado eficiencias de $59.4 \% \mathrm{y}$ $57.7 \%$, en el primer y segundo año, respectivamente. Tales resultados son moderadamente elevados y caen en el rango de lo reportado en la literatura para sistemas similares (Vymazal 2013). Los probables mecanismos responsables de estas eficiencias elevadas fueron, en la primera etapa la remoción de $\mathrm{NO}_{3}{ }^{-}$vía desnitrificación parcial, la captación de $\mathrm{NO}_{3}{ }^{-}$y $\mathrm{NH}_{4}{ }^{+}$por las plantas emergentes, así como la adsorción del $\mathrm{NH}_{4}{ }^{+}$en el medio filtrante. Mientras que, en la segunda etapa, el mecanismo predominante fue la volatilización del $\mathrm{NH}_{4}{ }^{+}$. En contraste, los sistemas II (HSSFH-HSSFV) y III (HSSFV-HSSFH) fueron menos efectivos y sin diferencia entre ellos, con eficiencias de $21.2 \%$ y $25.0 \%$, respectivamente en el primer año y $34.4 \%$, y $36.3 \%$, respectivamente en el segundo año. Se evidenció una clara tendencia hacia una mayor eficiencia en el segundo periodo (incremento significativo para el sistema II). Tales resultados, caen nuevamente dentro de los rangos reportados en la literatura para sistemas híbridos HSSFH-HSSFV o viceversa (Vymazal 2013). La mayor eficiencia del sistema I coincide con lo reportado por Vymazal (2013) quien encontró que los humedales híbridos que incluían humedales superficiales, similares a las LE, son los más efectivos debido a la volatilización de $\mathrm{N}-\mathrm{NH}_{4}{ }^{+}$ante la elevación de $\mathrm{pH}$ ocasionada por la actividad fotosintética de las algas y la vegetación sumergida, así como la desnitrificación que puede tener lugar en los sedimentos del fondo.

Por otra parte, es importante resaltar que los tres sistemas híbridos evaluados fueron bastante efectivos para la nitrificación. Sin embargo, el proceso nitrificante por sí sólo no remueve el nitrógeno de las aguas residuales. Para lograr la remoción, se requiere que la desnitrificación se lleve a cabo en el sistema. Este proceso, conocido como desnitrificación heterótrofa se presenta en ausencia de oxígeno o bien en cantidades relativamente muy pequeñas, dando lugar al potencial de reducción-oxidación (redox) de $+50 \mathrm{mV}$ a $-50 \mathrm{mV}$. En dichas condiciones y en presencia de materia orgánica, ocurre la transformación biológica del $\mathrm{NO}_{3}{ }^{-} \mathrm{a}$ óxidos de nitrógeno y finalmente a $\mathrm{N}_{2}$ (Gerardi 2002). En el sistema II (similar al sistema I), la desnitrificación sólo tuvo lugar en la primera etapa y no de manera completa. Algunos factores que, probablemente, evitaron una mayor desnitrificación fueron la corta altura de los HSSFH así como la utilización de un medio filtrante fino. Otros procesos que coadyuvaron en la remoción de $\mathrm{N}_{\text {total }}$ fueron la captación por las plantas y la adsorción del $\mathrm{N}_{-} \mathrm{NH}_{4}{ }^{+}$en el sustrato y raíces (Sun et al. 2005, Gutiérrez-Mosquera y Peña-Varón 2011, Tee et al. 2012). En la segunda etapa de este sistema, la remoción de $\mathrm{N}_{\text {total }}$ fue nula debido a que el mecanismo predominante fue la transformación del $\mathrm{NH}_{4}{ }^{+}$ a $\mathrm{NO}_{3}{ }^{-}$ya que en estas condiciones, la desnitrificación no puede llevarse a cabo. Respecto al sistema III, en la primera etapa, tuvo lugar la nitrificación de manera eficiente. Sin embargo, la desnitrificación heterótrofa esperada en la segunda etapa (HSSFH) no fue posible, nuevamente debido a las características del humedal, al agotamiento de la materia orgánica durante el paso del agua residual en los HSSFV (ubicados como primera etapa), así como a la prevalencia de condiciones oxidadas y potenciales redox positivos por la descarga directa del efluente procedente de los HSSFV (Tejeda et al. 2015), tales condiciones se incrementaron probablemente, por el oxígeno suministrado por la $C$. indica, ante una cobertura total, sobre todo en el segundo año. Tee et al. (2012) encontraron potenciales redox significativamente más altos en HSSFH de $0.30 \mathrm{~m}$ al compararlos con sistemas de $0.60 \mathrm{~cm}$. Asimismo, estos autores encontraron una mayor nitrificación correlacionada con potenciales redox más positivos en los sistemas de menor profundidad. De esta forma, en ausencia de la desnitrificación, los mecanismos que intervinieron en la remoción de $\mathrm{N}_{\text {total }}$ en la segunda etapa, probablemente fueron los mismos que se discutieron para la primera etapa de los sistemas I y II.

Respecto a los humedales ubicados como primera etapa, conforme a lo esperado, se encontró que los HSSFH fueron más efectivos que los HSSFV para la remoción de $\mathrm{N}_{\text {total }}$ en los dos periodos de evaluación $(\mathrm{p}<0.05)$. De acuerdo con algunos autores (Cui et al. 2010), la porosidad del sustrato es determinante para que pueda tener lugar la desnitrificación en los HSSFV, si es un material con una porosidad elevada (como en este caso, el tezontle), se facilita la aireación por lo que se inhibe la desnitrificación.

\section{CONCLUSIONES}

Los tres sistemas de humedales estudiados, fueron efectivos para la nitrificación del $\mathrm{N}_{-} \mathrm{NH}_{4}{ }^{+}$, por lo que 
las remociones del $\mathrm{N}_{-} \mathrm{NH}_{4}{ }^{+}$variaron entre el $76 \mathrm{y}$ el $87 \%$ en el primer año, y entre el 79 y el $97 \%$ en el segundo. Sin embargo, fueron poco efectivos para la desnitrificación, ya que se obtuvieron efluentes altamente nitrificados. La mayor remoción de $\mathrm{N}_{\text {total }}$ se logró en el sistema I, como resultado de la presencia de LE que probablemente permitió la volatilización de $\mathrm{N}-\mathrm{NH}_{3}$ ante los $\mathrm{pH}$ generados por la actividad fotosintética de las algas, además de una posible desnitrificación en los sedimentos. Por el contrario, en los otros sistemas II y III la remoción de $\mathrm{N}_{\text {total }}$ fue baja $(21 \% / 34 \%$ y $25 \% / 36 \%$, respectivamente), debido probablemente, a factores como el tamaño fino del tezontle empleado, así como su elevada porosidad en comparación con otros medios filtrantes comunes en humedales de tratamiento, aunado a la poca profundidad de los HSSFH. Por lo que dichas condiciones no favorecieron la creación de amplias zonas anóxicas/anaerobias.

Por otra parte, se observó una clara tendencia en los dos tipos de humedales hacia una mayor eficiencia en el segundo año, posiblemente derivado de una mayor influencia de la vegetación emergente en los mecanismos de transformación/remoción de los componentes nitrogenados.

\section{REFERENCIAS}

APHA, AWWA, WEF (2005). Standard methods for the examination of water and wastewater. 21a ed. American Public Health Association - American Water Works Association - Water Environment Federation, Washington, DC, EUA, 1368 pp.

Ayaz S. C., Aktas Ö., Akca L. y Findik N. (2015). Effluent quality and reuse potential of domestic wastewater treated in a pilot-scale hybrid constructed wetland system. J. Environ. Manage. 156, 115-120. DOI: 10.1016/j.jenvman.2015.03.042

Brix H., Arias C. y Johansen N.H. (2003). Experiments in a two stage constructed wetland system: nitrification capacity and effects of recycling on nitrogen removal. En: Wetlands: Nutrients, metals and mass cycling. (J. Vymazal, Ed.). Backhuys Publishers, Leiden, Holanda, pp. 237-258.

Buss R., Herbert A.W., Morgan P., Thornton S.F. y Smith J.W.N. (2004). A review of ammonium attenuation in soil and groundwater. Q. J. Eng. Geol. Hydroge. 37, 347-359. DOI: 10.1144/1470-9236/04-005

Cui L., Ouyang Y., Lou Q., Yang F., Chen Y., Zhu W. y Luo S. (2010). Removal of nutrients from wastewater with Canna indica L. under different vertical-flow constructed wetland conditions. Ecol. Eng. 36, 10831088. DOI: $10.1016 /$ j.ecoleng.2010.04.026
Ding Y., Wang W., Song X. S. y Wang Y. H. (2014). Spatial distribution characteristics of environmental parameters and nitrogenous compounds in horizontal subsurface flow constructed wetland treating high nitrogen-content wastewater. Ecol. Eng., 70, 446-449. DOI: 10.1016/j.ecoleng.2014.06.008

Gerardi M.H. (2002). Nitrification and denitrification in the activated sludge process. John Wiley and Sons, Inc., Nueva York, EUA, 193 pp.

Gutiérrez-Mosquera H. y Peña-Varón M. R. (2011). Eliminación de nitrógeno en un humedal construido subsuperficial, plantado con Heliconia psíttacorum. Tecnología y Ciencias del Agua 2, 49-60.

Kadlec R. H. y Knight R. L. (1996). Treatment wetlands. CRC Press, Florida, EUA, 928 pp.

Langergraber G., Prandtstetten Ch., Pressl A., Sleytr K., Leroch K., Rohrhofer R. y Haberl R. (2008). Investigations on nitrogen removal in a two-stage subsurface vertical flow constructed cetland, Capítulo 18. En: Wastewater treatment, plant dynamics and management in constructed and natural wetlands. (J. Vymazal, Ed.). Springer Science and Business Media. Třeboň, República Checa, pp. 199-209.

DOI: $10.1007 / 978-1-4020-8235-118$

Mitsch W. J. y Gosselink J. G. (2007). Wetlands, 4a ed. John Wiley and Sons, Inc., Nueva York, EUA, 582 pp.

Masi F. y Martinuzzi N. (2007). Constructed wetlands for the Mediterranean countries: hybrid systems for water reuse and sustainable sanitation. Desalination 215, 4455. DOI: $10.1016 /$ j.desal.2006.11.014

Metcalf y Eddy (2003). Wastewater engineering treatment and reuse, 4a. ed. McGraw-Hill, Nueva York, EUA, $1819 \mathrm{pp}$.

Morató J., Codony F., Sánchez O., Pérez L. M., García J. y Mas J. (2014). Key design factors affecting microbial community composition and pathogenic organism removal in horizontal subsurface flow constructed wetlands. Sci. Total Environ. 481, 81-89.

DOI: $10.1016 /$ j.scitotenv.2014.01.068

Paul E.A. y Clark F.E. (1989). Soil microbiology and biochemistry, 1a. ed. Academic Press, San Diego, California, EUA, 273 pp.

Park J. B .K., Craggs R. J. y Shilton A. N. (2011). Wastewater treatment high rate algal ponds for biofuel production. Bioresour. Technol. 102, 35-42.

DOI: 10.1016/j.biortech.2010.06.158

Rawat I., Ranjith Kumar R., Mutanda T. y Bux F. (2011). Dual role of microalgae: Phycoremediation of domestic wastewater and biomass production for sustainable biofuels production. Appl. Energ. 88, 3411-3424. DOI: 10.1016/j.apenergy.2010.11.025

Rivas A., Barceló-Quintal I. y Moeller G. E. (2011) Pollutant removal in a multi-stage municipal wastewater 
treatment system comprised of constructed wetlands and a maduration pond, in a temperate climate. Water Sci. Technol. 64, 980-987. DOI: 10.2166/wst.2011.731

Sun G., Zhao Y. y Allen S. (2005). Enhanced removal of organic matter and ammoniacal-nitrogen in a column experimental of tidal flow constructed wetland system. J. Biotechnol. 115, 189-197. DOI: 10.1016/j.jbiotec.2004.08.009

Tee H. C., Lim P. E., Seng C. E. y Nawi M. (2012). Newly developed baffled subsurface-flow constructed wetland for the enhancement of nitrogen removal. Biores. Technol. 104, 235-242.

DOI: 10.1016/j.biortech.2011.11.032

Tejeda A., López Z., Rojas D., Reyna M., Barrera A. y Zurita F. (2015). Eficiencia de tres sistemas de humedales híbridos para la remoción de carbamazepina. Tecnología y Ciencias del Agua 6, 19-31.

Vymazal J. (2005). Horizontal sub-surface flow and hybrid constructed wetlands systems for wastewater treatment. Ecol. Eng. 25, 478-490.

DOI: 10.1016/j.ecoleng.2005.07.010

Vymazal J. (2007). Removal of nutrients in various types of constructed wetlands. Sci. Tot. Environ. 380, 48-65. DOI: 10.1016/j.scitotenv.2006.09.014
Vymazal J. (2013). The use of hybrid constructed wetlands for wastewater treatment with special attention to nitrogen removal: A review of a recent development. Water Res. 47, 4795-4811. DOI: 10.1016/j.watres.2013.05.029

Vymazal J. y Kropfelová L. (2008). Wastewater treatment in constructed wetlands with horizontal sub-surface flow. 1a. ed. Springer Science and Business Media, Dordrecht, Holanda, 566 pp.

DOI: $10.1007 / 978-1-4020-8580-2$

Vymazal J. y Kropfelová L. (2011). A three-stage experimental constructed wetland for treatment of domestic sewage: First 2 years of operation. Ecol. Eng. 37, 9098. DOI: 10.1016/j.ecoleng.2010.03.004

Zurita F. y White J. R. (2014). Comparative study of three two-stage hybrid ecological wastewater treatment systems for producing high nutrient, reclaimed water for irrigation reuse in developing countries. Water 6, 213-228. DOI: 10.3390/w6020213

Zurita F., De Anda J. y Belmont M. A. (2009). Treatment of domestic wastewater and production of commercial flowers in vertical and horizontal subsurface-flow constructed wetlands. Ecol. Eng. 35, 861-869. DOI: $10.1016 /$ j.ecoleng.2008.12.026 